\title{
Asymptomatic carriage of Haemophilus ducreyi confirmed by the polymerase chain reaction
}

\author{
S Hawkes, B West, S Wilson, H Whittle, D Mabey
}

\begin{abstract}
Objective-To investigate asymptomatic carriage of Haemophilus ducreyi by using polymerase chain reaction (PCR) on samples from women at high risk of infection.

Subjects-213 commercial sex workers (CSWs) recruited in The Gambia, West Africa.

Methods-Genital samples (cervical, vaginal and ulcer) were tested for the presence of $H$ ducreyi by PCR with the technique of "one tube nested primer". Results-12 CSWs were PCR positive for $H$ ducreyi; 8 of these women had genital ulcers on examination.

Conclusion-Using a simplified PCR technique for detecting $H$ ducreyi we have shown that $2 \%$ of CSWs were carrying the organism without clinical symptoms or signs. This has important implications for sexually transmitted disease control programmes in areas with a high prevalence of chancroid.
\end{abstract}

(Genitourin Med 1995;71:224-227)

Keywords: Haemophilus ducreyi; Asymptomatic carriage, $\mathrm{PCR}$

\section{Introduction}

The control of all sexually transmitted diseases (STDs) has assumed a greater priority in public health campaigns throughout the world since the association between a history of STDs and acquisition of HIV infection was noted. ${ }^{12}$ The strongest association is found in those with a history of genital ulceration secondary to a sexually transmitted pathogen. ${ }^{3}$

Genital ulcer disease is prevalent throughout the world, but its aetiology depends on the geographical location. ${ }^{4}$ In the industrialised world Haemophilus ducreyi as a cause of genital ulceration is uncommon, although sporadic outbreaks of chancroid have been reported especially in North America, ${ }^{5}$ where a recent rise in incidence is thought to be related to an increase in selling sex in exchange for drugs. ${ }^{6}$ Previous studies in Africa have shown that $H$ ducreyi is the most commonly diagnosed aetiological agent of genital ulceration. $^{4-9}$

In all countries where chancroid is endemic or epidemic there are common features in the epidemiology of the disease. It is predominantly diagnosed in men, the male:female ratio ranging from $3: 1$ to $25: 1$ or higher. ${ }^{5}$ It is often noted that the infected men cite commercial sex workers as their probable source of infection ${ }^{51011}$ and this may explain part of the difference in male/female diagnostic rates. It has been suggested that asymptomatic carriage of $H$ ducreyi, especially in women, may contribute to the continued spread of the infection. ${ }^{10}$

The exact methodology for achieving a sustainable decrease in STD incidence and prevalence has been widely debated. Two main approaches are currently favoured: a syndromic approach for the treatment of those with symptomatic disease and their partners; or case-finding through screening "at-risk" groups for the presence of any STDs (both symptomatic and asymptomatic) and treating them appropriately. Current guidelines from the World Health Organisation recommend treating any patient presenting with non-vesicular genital ulcers with antibiotics against syphilis and chancroid in the first instance. ${ }^{12}$ In practice many patients presenting for STD treatment will also have blood taken for syphilis serology. Thus, those with untreated, asymptomatic but infectious syphilis can be screened and treated within established health-care settings. However, this policy does not take into account any healthy individuals with asymptomatic carriage of potentially infectious $H$ ducreyi.

$H$ ducreyi is a fastidious organism requiring complex media and growth conditions, making culture difficult. ${ }^{13}$ Clinical diagnosis has a variable positive predictive value depending on the prevalence of chancroid in the community. ${ }^{14}$ Non cultural methods such as use of monoclonal or polyclonal antibodies in immunofluorescence tests and DNA probes have been developed. However, the newer DNA amplification methods using a polymerase chain reaction offer a more sensitive and specific approach to diagnosis. ${ }^{15} 16$

We report here the results from screening commercial sex workers in The Gambia for the presence of $H$ ducreyi, and the correlation between a PCR-positive diagnosis and clinical symptoms and signs.

\section{Methods}

Study population and study parameters

During four months in 1992/3, 213 self-identified female commercial sex workers in The Gambia were recruited into a study to measure their HIV seroprevalence, and correlation of HIV status with clinical symptoms and laboratory diagnosis of other STDs. All 
women underwent serological testing for HIV. Six women declined a clinical examination as they were menstruating at the time of their initial visit and did not return for a second visit. Sera from all women were also tested using commercially available kits for rapid plasma reagin (RPR, Wellcome VD-25) and Treponema pallidum haemagglutination assay (TPHA, Fujirebio).

The remaining 207 consenting women underwent a full clinical examination including a pelvic and speculum examination. During the speculum examination, a dry sterile swab was taken from the cervix, posterior fornix and the base of an ulcer if present (ulcers were not cleaned before sampling). This swab was then placed in $0.5 \mathrm{ml}$ phosphate buffered saline and stored immediately at $-70^{\circ} \mathrm{C}$. All frozen samples were then transported back to England, for PCR. We also took a cervical swab for Chlamydia trachomatis antigen detection by enzyme immunoassay (IDEIA, Dako diagnostics, Ely UK), a cervical and a vaginal swab for Gram staining and a cervical PAP smear to look for evidence of cervical dysplasia. There were no local facilities for culturing herpes simplex virus or Neisseria gonorrhoeae; diagnosis of the latter relied upon Gram staining.

In order to confirm the specificity of the PCR assay, cervical swabs were taken from 50 antenatal clinic attenders at University College Hospital, London. These women were unlikely to have been exposed to $H$ ducreyi.

\section{PCR techniques}

Processing of samples. Contamination of new reactions with previously amplified products leading to false positive reactions is a persistent problem with the PCR technique and PCR studies. To guard against contamination physical separation of the work areas as described in Wilson et $a l^{17}$ was employed. In addition samples were divided and processed in duplicate to give a measure of the degree or rate of contamination which was present in this series of experiments, and 40 negative controls, consisting of PBS alone, were processed in the same way as clinical specimens and were interspersed among the latter.

Swabs were defrosted, and contents expelled into the suspending buffer which was divided for duplicate processing, PCR and analysis. The samples were centrifuged at $12,000 \mathrm{~g}$ for 5 minutes, the supernatant discarded and the pellet resuspended in $1 \mathrm{ml}$ of $50 \mathrm{mM}$ Tris buffer, $\mathrm{pH} 8 \cdot 3$. After spinning again at $12,000 \mathrm{~g}$ for 5 minutes, the supernatant was discarded and the pellet resuspended in $50 \mu \mathrm{l}$ of Tris buffer. Samples were then heated at $80^{\circ} \mathrm{C}$ for 30 minutes. After adding $50 \mu \mathrm{l}$ of chloroform, the sample was mixed well and spun at $12,000 \mathrm{~g}$ for $2 \mathrm{~min}$ utes. The upper aqueous phase $(5 \mu \mathrm{l})$ containing the nucleic acids was used in a $20 \mu \mathrm{l} \mathrm{PCR}$. Samples were stored at $-20^{\circ} \mathrm{C}$ without removing the chloroform and were recentrifuged prior to use after storage.

Polymerase chain reaction. We used the tech- nique of "one-tube nested PCR" as described by Wilson et al. ${ }^{17}$ The PCR target was the $16 \mathrm{~S}$ rRNA gene of $H$ ducreyi and primers were taken from sequences published by Rossau et $a{ }^{18}$

The outer primers used were HD996 (5'TGTGACAGGTGCTGCATGGCTGTC) and HD 1400 (3'-GCGATTACTAGCGATTCCGACTTCA). The inner primers were HD 1279 (3'-GTCGAGTTGCAGACTC) and HD1078 (5'-ATGTAA/GTGATGGAAC). The inner primers, HD1279 and HD1078, were also labelled at their $5^{\circ}$ ends with biotin and digoxigenin respectively during their commercial synthesis ( $R$ and $D$ Systems, Europe Limited). Primers were used at a 0.6 pmoles of each outer primer, 10 pmoles of each inner primer per reaction. To minimise costs, $2 / 3$ of each inner primer in the reaction was used unlabelled. The reaction contained 5\% DMSO. The "hot start" method of PCR was used to minimise primerdimer formation. The reaction conditions and cycling parameters were identical to those described by Wilson et al. ${ }^{17}$

Colorimetric detection of PCR product The method used was again identical to that described by Wilson et al, ${ }^{17}$ except that 3\% milk powder was included in the antibody incubation step.

Analysis of results Only those samples which were positive or negative in duplicate were classified as such. PCR was repeated on samples which were discordant. The numbers of samples in which the duplicates did not match and which were subsequently found to be negative on repeat PCR were noted as this is a measure of the rate of contamination in this study. ${ }^{17}$

\section{Results}

Specificity of the PCR

Forty negative controls were all negative by PCR, and samples from all 50 antenatal clinic attenders in London were also negative. Ten duplicates from Gambian subjects did not match; these were all negative in both duplicates on repeat testing. Thus the rate of contamination of new reactions with previously amplified products was 10 out of the total number of negative reactions $(n=390)$. The chance of any one sample being accidentally contaminated by PCR products in both duplicates halves was therefore $10 / 390 \times 10 / 390=$ $1 / 2321$. Thus in an analysis of more than 2000 samples only one false positive would have been generated.

\section{Samples from commercial sex workers in the Gambia}

Of the 213 women recruited to the study, 207 had a full clinical examination. Eighteen women were diagnosed as having genital ulcers on clinical examination: eight of these women were PCR positive for $H$ ducreyi. PCR was also positive for $H$ ducreyi in a further four women who had no clinical signs of genital ulcer disease. Of the 18 women with genital ulcers, five were TPHA and RPR positive 
(one $H$ ducreyi positive also), and eight were HIV-positive (five with $H$ ducreyr). Five women with genital ulcers had no evidence of $H$ ducreyi, and no serological evidence of syphilis or HIV.

$C$ trachomatis antigen was detected in eight women (4\%) and 25 (12\%) had intracellular diplococci noted on cervical Gram stains indicating infection with $N$ gonorrhoeae. One woman with gonorrhoea was also PCR positive for $H$ ducreyi. Overall, 74 (35\%) women were HIV positive on serological testing: 17 (8\%) were HIV-1 positive, 45 (21\%) were HIV-2 positive and $12(6 \%)$ were dually reactive to both HIV-1 and HIV-2. In addition, 47 (22\%) women were both TPHA and RPR positive, whilst a further $94(45 \%)$ were solely TPHA positive. Five $(42 \%)$ of the 12 women who had $H$ ducreyi diagnosed on PCR were HIV antibody positive on serological testing. Four of the five were HIV-2 positive and one woman had serum which was dually reactive.

Women with $H$ ducreyi did not present more frequently with other symptoms or signs. One woman with $H$ ducreyi infection and genital ulceration was noted to have adnexal tenderness on examination, but she was found to have a concomitant infection with Trichomonas vaginalis. Likewise, only one woman had an abnormal vaginal discharge-a lower prevalence of this finding in comparison with women who were PCR negative for $H$ ducreyi.

\section{Discussion}

The importance of treating and controlling STDs must be emphasised. Not only are there immediate beneficial effects to the individual and his/her sexual partner(s), but there is substantial evidence that STDs, especially those causing genital ulceration, play a major role in facilitating the transmission of HIV. ${ }^{1}$ Indeed, previous surveys in The Gambia have found that HIV seropositivity is associated with serological evidence of genital ulcer disease in both commercial sex workers and male STD clinic attenders. ${ }^{19} 20$ The World Bank notes that in countries where HIV incidence is high, and STDs are endemic, treatment of STDs is a highly cost-effective method of reducing transmission of HIV. ${ }^{21}$

STD control is, however, severely hampered by the fact that infections are often asymptomatic, especially in women. Chlamydia trachomatis, for example, probably only causes overt symptoms in one third of women harbouring the infection. ${ }^{22}$ The existence of a 'reservoir' of asymptomatic carriers of $H$ ducreyi, however, has only been demonstrated in one previous study from Africa. ${ }^{10}$ Nonetheless, asymptomatic carriage of $H$ ducreyi in women is considered to be a contributory factor in the continued spread of the infection.

We have used PCR techniques to demonstrate that $2 \%(4 / 207)$ of commercial sex workers in our study were carrying $H$ ducreyi asymptomatically. These women had no evidence of genital ulceration, and no other symptoms or signs of infection such as inguinal buboes. This rate of asymptomatic carriage is similar to the $4 \%$ found in CSWs in Nairobi by Plummer et al in 1983. ${ }^{10}$

Despite precautions contamination is a persistent problem in PCR studies and is often overlooked. In this study we were able to monitor the rate of contamination and to calculate the likelihood of a sample being false positive in both duplicates. We are satisfied that the rate of contamination in this study was very low and it is extremely unlikely that the PCR positive patients identified in this study were due to contamination. Moreover, 50 swabs collected from antenatal clinic attenders in London were negative.

The PCR technique used in this study was developed as a simplified procedure with chloroform extraction of DNA, a one tube nested PCR and a colour reaction for detection. Previous studies evaluating this method on clinical samples from four different geographical regions in Africa have yielded a sensitivity of $83 \%$ when compared with clinical diagnosis, whilst culture on the same samples had a sensitivity of $77 \%$. PCR compared with culture gave a sensitivity $94 \% .{ }^{23}$ This technique did not give any false positives when tested against other strains of closely related bacteria or common contaminants of the genital tract.

The method was designed to be used in areas where culture facilities are unreliable or do not exist, but where new technology is affordable. Procedures are simplified by using the minimum of manipulation, hence lowering chances of contamination. Without such a technique it would be more difficult to carry out epidemiological studies on the prevalence of $H$ ducreyi.

It is possible that the women in our study were in the incubation period of chancroid, although this would seem unlikely to explain all asymptomatic carriage as the incubation period is believed to be short (3-7 days) ${ }^{24} \mathrm{~A}$ second possibility is that these women had partially treated chancroid, and although they no longer had genital ulcers they were still harbouring the organism. More than half $(51 \% ; 108 / 213)$ of the women who participated in the study were taking antibiotics regularly $-75 \%$ of these $(81 / 108)$ were taking an antibiotic (usually at sub-therapeutic doses) every day. Notably, three of the four women with asymptomatic carriage of $H$ ducreyi were taking antibiotics every day compared to only one woman with $H$ ducreyi and a genital ulcer. However, the antibiotics most commonly used were tetracycline and ampicillin, both of which have been shown to be ineffective against $H$ ducreyi in many areas including The Gambia $^{25}$ since the emergence of plasmidmediated resistance in the late 1970 s. $^{26}$

Crucially, it is not known whether these women are infectious to their sexual partners, and if the presence of $H$ ducreyi without genital ulceration has any effect on the transmissibility of HIV between partners. Further prospective cohort studies are needed to assess the true importance of asymptomatic carriage of this organism. 
This work was generously supported by the Overseas Development Administration. We acknowledge the help of Ramou Jagne in The Gambia, and Mr Tim Mould at University College Hospital, London.

1 Wasserheit JN. Epidemiological synergy: interrelationships between HIV infection and other STDs. Sex Transm Dis 1992;19:61-77.

1992;19:61-77.
2 Moses S, Plummer FA, Ngugi EN, Nagelkerke NJD, Anzala AO, NdinyaAchola JO. Controlling HIV in Africa: Effectiveness and cost of an intervention in a high-frequency STD transmitter core group. AIDS 1991;5:407-11.

3 Cameron DW, D'Costa LJ, Maitha GM, et al. Female to male transmission of HIV-1: risk factors for seroconversion in men. Lancet 1989;2:403-7.

4 Morse SA, Chancroid and Haemophilus ducreyi. Clin Microbiol Rev 1989;2:137-57.

5 Schmid GP, Sanders LL, Blount JH, Alexander ER. Chancroid in the US: reestablishment of an old disease. FAMA 1987;258:3265-8.

6 Martin DH, DiCarlo RP. Recent changes in the epidemiology of genital ulcer disease in the USA: The crack ogy of genital ulcer disease in the USA: The crack
cocaine connection. Sex Transm Dis 1994;21(S2):S76cocaine

7 Mabey DCW, Wall RA, Bello CSS. Aetiology of genital ulceration in The Gambia. Genitourin Med 1987;63: 312-5.

8 Bogaerts J, Alvarez Ricart C, van Dyck E, Piot P. The etiology of genital ulceration in Rwanda. Sex Transm Dis 1989;3:123-6.

9 Dangor Y, Fehler G, Exposto FDLMPP, Koornhof HJ. Causes and treatment of sexually acquired genital ulceration in Southern Africa. $S$ Af Med $\mathcal{f} 1987 ; 76$ : ulceration

10 Plummer FA, Nsanze H, Karasira, D'Costa LJ, Dylewski J, Ronald AR. Epidemiology of chancroid and Haemophilus Ronald AR. Epidemiology of chancroid and Haem
ducreyi in Nairobi, Kenya. Lancet 1983;ii:1293-5.

11 Blackmore CA, Limpakaenjanarat K, Rigau-Perez JG Albritton WL, Greenwood JR. An outbreak of chancroid in Orange County, California: descriptive epidemiology and control measures. $\mathcal{F}$ Inf $D$ is 1985;151:840-4.

12 WHO Study Group. Management of Patients with Sexually Transmitted Diseases. WHO Technical Report Series, Report No. 810. 1991.
13 Jones CC, Rosen T. Cultural diagnosis of chancroid. Arch Dermatol 1991;127:1823-7.

14 Dangor Y, Ballard RC, Da L'Exposto F, et al. Accuracy of clinical diagnosis of genital ulcer disease. Sex Transm Dis 1990;17:184-9.

15 Chui L, Albritton W, Paster B, Maclean I, Marusyk R. Development of the polymerase chain reaction for diagnosis of chancroid. F Clin Micro 1993;31:659-64.

16 Johnson SR, Martin DH, Cammarata C, Morse SA Development of a polymerase chain reaction assay for Development of a polymerase chain reaction assay for
the detection of Haemophilus ducreyi. Sex Transm Dis. the detection of

17 Wilson SM, McNerny R, Nye PM, Godfrey-Faussett PD, Stoker NG, Voller A. Progress towards a simplified polymerase chain reaction and its application to diagnosis of tuberculosis. F Clin Microbiol 1993;31:776-82.

18 Rossau R, Duhamel G, Jannes G, Decourt J, Heuverswyn $H$. The development of specific rRNA-derived oligonucleotide probes for Haemophilus ducreyi, the causative organism of chancroid. $\mathcal{F}$ Gen Microbiol 1991;137: 277-85.

19 Pepin J, Quigley M, Todd J, et al. Association between HIV-2 infection and genital ulcer disease among male STD patients in The Gambia. AIDS 1992;6:489-3.

20 Pepin J, Dunn D, Gaye I, et al. HIV-2 infection among prostitutes working in The Gambia: Association with serological evidence of genital ulcer disease and with generalized lymphadenopathy. AIDS 1991;5:69-75.

21 World Development Report 1993. Investing in Health. World Bank, Oxford University Press 1993.

22 Stamm WE, Holmes KK. Chlamydia trachomatis infections of the adult. In: Holmes KK, Mårdh P-A, Sparling PF, et al. eds. Sexually Transmitted Diseases. 1990 New York: McGraw Hill.

23 West B, Wilson S, Changalucha J, et al. A simplified PCR for the detection of Haemophilus ducreyi and diagnosis of chancroid. F Clin Microbiol 1995;33:787-90.

24 Ronald AR, Albritton W. Chancroid and Haemophilus ducreyi. In: Holmes KK, Mårdh P-A, Sparling PF, ducreyi. In: Holmes KK, Mårdh P-A, Sparling PF, et al eds. Sexualh

25 Wall RA, Mabey DCW, Bello CSS, Felmingham D. The comparative in-vitro activity of 12 4-quinolone antimicrobials against Haemophilus ducreyi. $\mathcal{f}$ Antimicrob Chemother 1985;16:165-8.

26 McNicol PJ, Ronald AR. The plasmids of Haemophilus ducreyi. F Antimicrob Chemother 1984;14:561-3. 